\title{
Some Spectral Properties of p-Laplacian Diffusion Boundary Value Problem on Time Scales
}

\author{
Tuba Gulsen ${ }^{1,}$, Emrah Yilmaz ${ }^{1}$, and Meltem Kayall ${ }^{1}$ \\ ${ }^{1}$ Department of Mathematics, Firat University, Elazig, Turkey
}

\begin{abstract}
In this paper, we consider $p$-Laplacian type Diffusion boundary value problem on an arbitrary time scale. We generalize some spectral properties of $p$-Laplacian Diffusion problem to an arbitrary time scale. Moreover, we prove Picone's identity for this problem.
\end{abstract}

\section{Introduction}

The theory of time scale calculus was introduced for the first time by Stefan Hilger in his Ph.D. thesis in 1988 [1]. A time scale $\mathbb{T}$ is an arbitrary, non-empty, closed subset of $\mathbb{R}$. For standard notions and notations connected to time scale calculus, we refer to [2].

The significance of working boundary value problems on time scales was understood and some important results were obtained. In 2002, Agarwal et al. [3] studied linear dynamic equations and initial value problems on time scales. Guseinov and Kaymakçalan [4] considered second order linear dynamic equations and gave some sufficient conditions for nonoscillation on time scales in 2002. Erbe et al. [5] gave several comparison theorems for second order linear dynamic equation on time scales. In 2003, Guseinov [6] investigated Riemann and Lebesgue integration on time scales and gave the fundamental theorems of calculus. In 2007, Rynne [7] defined space of square-integrable functions and Sobolev type spaces on time scales and gave practices of the functional analytic results to SturmLiouville type boundary value operator. Grace et al. [8] studied second order half-linear dynamic equations on time scales and investigated some oscillation criteria in 2009.

In recent years, several authors have obtained many important results about $p$-Laplacian boundary value problem on time scales (see [9-12]). Anderson et al. [13] proved that there is at least one positive solution for a one dimensional $p$-Laplacian delta-nabla dynamic equation on time scales in 2004. He [14] proved that there exists at least double positive solutions with three-point boundary conditions for $p$-Laplacian dynamic equation on time scales in 2005. Sun and Li [15] considered a one dimensional $p$ - Laplacian boundary value problem and showed existence of this problem on time scales in 2007. Binding et al. [16] considered $p$-Laplacian nonlinear eigenvalue problem on a half line in 2010. Su and

*Corresponding author: tubagulsen87@hotmail.com 
Feng [17] studied a second-order $p$-Laplacian dynamic boundary value problem on a periodic time scale and showed existence of solutions of this problem in 2017.

In this study, we want to prove some basic spectral properties of $p$-Laplacian Diffusion eigenvalue problem of the form

$$
L y^{(p-1)}=-\left(y^{\Delta(p-1)}\right)^{\Delta}(t)+(p-1)\{q(t)+2 \lambda r(t)\} y^{\sigma(p-1)}(t)=\lambda^{2}(p-1) y^{\sigma(p-1)}(t),
$$

with the separated boundary conditions

$$
\begin{gathered}
y^{\Delta(p-1)}(\rho(a))=0, \\
\alpha y^{(p-1)}(b)+\beta y^{\Delta(p-1)}(b)=0,
\end{gathered}
$$

where $\lambda$ is a spectral parameter and $t \in[\rho(a), b] \cap \mathbb{T}$. During this study, we suppose that $q, r \in L_{2}^{\Delta}[\rho(a), b]$ are continuous functions ; $p>1 ; a, b \in \mathbb{T}$ with $y^{\sigma(p-1)}=y^{(p-1)}(\sigma)$, $a<b,\left(\alpha^{2}+\beta^{2}\right) \neq 0 . y^{(p-1)} \in C_{r d}^{2}([\rho(a), b], \mathbb{R})$ is eigenfunction of the problem (1)-(3). By setting $\mathbb{T}=\mathbb{R}$ in (1), we get below classical $p$ - Laplacian Diffusion equation

$$
-\left(y^{(p-1)}\right)^{\prime}(t)=(p-1)\left(\lambda^{2}-q(t)-2 \lambda r(t)\right) y^{(p-1)}(t)
$$

For $p=2$ in (4), we have

$$
-y^{\prime \prime}(t)+(q(t)+2 \lambda r(t)) y(t)=\lambda^{2} y(t), t \in[a, b] .
$$

(5) is known as classical Diffusion equation (or quadratic pencil of differential pencil) in literature. There are numerous studies in spectral theory related to this equation. The studies of $p$ - Laplacian type Diffusion equation is as follows. In [18], Koyunbakan considered the inverse problem for $p$-Laplacian Schrödinger equation with energy-dependent potential function and found asymptotic formulas of eigenvalues, nodal points and nodal lenghts by using Prüfer substitution in 2013. In [19], Gulsen and Yilmaz obtained asymptotic formulas of eigenvalues and nodal parameters and reconstructed the potential function $p$-Laplacian Diffusion problem in 2016. In [20], Gulsen et al. took into consideration Diffusion and impulsive Diffusion eigenvalue problems, respectively and showed some spectral properties of these problems on time scales. This study is organized as follows: In Section 2 , we prove some basic theorems and Picone's identity for the $p$-Laplacian Diffusion equation on $\mathbb{T}$. In Section 3, we give a conclusion to summarize our study.

\section{Main Results}

In this section, we give some valuable results for $p$-Laplacian Diffusion problem on a time scale $\mathbb{T}$ whose all points are right dense. It is well known that the problem (1)-(3) has only real, simple eigenvalues and eigenfunctions which are orthogonal to each other for $\mathbb{T}=\mathbb{R}[21]$. The following results will generalize these basic results.

Theorem 1. The eigenvalues of the problem (1)-(3) are all simple.

Theorem 2. Let $y_{1}^{(p-1)}, y_{2}^{(p-1)} \in C_{r d}^{2}([\rho(a), b], \mathbb{R})$ be the eigenfunctions of the problem (1)(3). Then, below equalities hold.

a) $\left(L y_{1}^{(p-1)}\right) y_{2}^{\sigma(p-1)}-\left(L y_{2}^{(p-1)}\right) y_{1}^{\sigma(p-1)}=W^{\Delta}\left(y_{1}^{(p-1)}, y_{2}^{(p-1)}\right)$, on $[\rho(a), b] \cap \mathbb{T}$.

b) $<L y_{1}^{(p-1)}, y_{2}^{\sigma(p-1)}>-<L y_{2}^{(p-1)}, y_{1}^{\sigma(p-1)}>=W\left(y_{1}^{(p-1)}, y_{2}^{(p-1)}\right)(b)-W\left(y_{1}^{(p-1)}, y_{2}^{(p-1)}\right)(\rho(a))$, where $W\left(y_{1}^{(p-1)}, y_{2}^{(p-1)}\right)=y_{1}^{(p-1)} y_{2}^{\Delta(p-1)}-y_{2}^{(p-1)} y_{1}^{\Delta(p-1)}$. 
Above theorems can be easily proved.

Theorem 3. The eigenfunctions $y_{1}^{(p-1)}\left(t, \lambda_{1}\right), y_{2}^{(p-1)}\left(t, \lambda_{2}\right)$ of the problem (1)-(3) corresponding to distinct eigenvalues $\lambda_{1}$ and $\lambda_{2}$, are orthogonal, i.e.

$$
(p-1)\left(\lambda_{1}+\lambda_{2}\right) \int_{\rho(a)}^{b} y_{1}^{\sigma(p-1)}\left(t, \lambda_{1}\right) y_{2}^{\sigma(p-1)}\left(t, \lambda_{2}\right) \Delta t-2(p-1) \int_{\rho(a)}^{b} r(t) y_{1}^{\sigma(p-1)}\left(t, \lambda_{1}\right) y_{2}^{\sigma(p-1)}\left(t, \lambda_{2}\right) \Delta t=0 .
$$

Proof. We have

$$
\begin{aligned}
& -\left(y_{1}^{\Delta(p-1)}\right)^{\Delta}(t)+(p-1)\left\{q(t)+2 \lambda_{1} r(t)\right\} y_{1}^{\sigma(p-1)}(t)=\lambda_{1}^{2}(p-1) y_{1}^{\sigma(p-1)}(t), \\
& -\left(y_{2}^{\Delta(p-1)}\right)^{\Delta}(t)+(p-1)\left\{q(t)+2 \lambda_{2} r(t)\right\} y_{2}^{\sigma(p-1)}(t)=\lambda_{2}^{2}(p-1) y_{2}^{\sigma(p-1)}(t) .
\end{aligned}
$$

Multiplying above equations by $y_{2}^{\sigma(p-1)}, y_{1}^{\sigma(p-1)}$, respectively, we have

$$
\begin{aligned}
& -\left(y_{1}^{\Delta(p-1)}\right)^{\Delta}(t) y_{2}^{\sigma(p-1)}(t)+(p-1)\left\{q(t)+2 \lambda_{1} r(t)\right\} y_{1}^{\sigma(p-1)}(t) y_{2}^{\sigma(p-1)}(t) \\
& =\lambda_{1}^{2}(p-1) y_{1}^{\sigma(p-1)}(t) y_{2}^{\sigma(p-1)}(t) \\
& -\left(y_{2}^{\Delta(p-1)}\right)^{\Delta}(t) y_{1}^{\sigma(p-1)}(t)+(p-1)\left\{q(t)+2 \lambda_{2} r(t)\right\} y_{2}^{\sigma(p-1)}(t) y_{1}^{\sigma(p-1)}(t) \\
& =\lambda_{2}^{2}(p-1) y_{2}^{\sigma(p-1)}(t) y_{1}^{\sigma(p-1)}(t) .
\end{aligned}
$$

Subtracting (6) by (7) leads to

$$
\begin{aligned}
& \left\{y_{2}^{(p-1)}\left(t, \lambda_{2}\right) y_{1}^{\Delta(p-1)}\left(t, \lambda_{1}\right)-y_{1}^{(p-1)}\left(t, \lambda_{1}\right) y_{2}^{\Delta(p-1)}\left(t, \lambda_{2}\right)\right\}^{\Delta} \\
& =(p-1)\left\{\left(\lambda_{2}^{2}-\lambda_{1}^{2}\right)-2\left(\lambda_{2}-\lambda_{1}\right) r(t)\right\} y_{1}^{\sigma(p-1)}\left(t, \lambda_{1}\right) y_{2}^{\sigma(p-1)}\left(t, \lambda_{2}\right) .
\end{aligned}
$$

Applying $\Delta$-integral to the last equality from $\rho(a)$ to $b$, we get

$$
\begin{aligned}
& \int_{\rho(a)}^{b}\left\{y_{2}^{(p-1)}\left(t, \lambda_{2}\right) y_{1}^{\Delta(p-1)}\left(t, \lambda_{1}\right)-y_{1}^{(p-1)}\left(t, \lambda_{1}\right) y_{2}^{\Delta(p-1)}\left(t, \lambda_{2}\right)\right\}^{\Delta} \Delta t \\
& =(p-1)\left(\lambda_{2}^{2}-\lambda_{1}^{2}\right) \int_{\rho(a)}^{b} y_{1}^{\sigma(p-1)}\left(t, \lambda_{1}\right) y_{2}^{\sigma(p-1)}\left(t, \lambda_{2}\right) \Delta t \\
& -2(p-1)\left(\lambda_{2}-\lambda_{1}\right) \int_{\rho(a)}^{b} r(t) y_{1}^{\sigma(p-1)}\left(t, \lambda_{1}\right) y_{2}^{\sigma(p-1)}\left(t, \lambda_{2}\right) \Delta t \\
& =-y_{1}^{(p-1)}\left(b, \lambda_{1}\right) y_{2}^{\Delta(p-1)}\left(b, \lambda_{2}\right)+y_{2}^{(p-1)}\left(b, \lambda_{2}\right) y_{1}^{\Delta(p-1)}\left(b, \lambda_{1}\right) \\
& +y_{1}^{(p-1)}\left(\rho(a), \lambda_{1}\right) y_{2}^{\Delta(p-1)}\left(\rho(a), \lambda_{2}\right)-y_{2}^{(p-1)}\left(\rho(a), \lambda_{2}\right) y_{1}^{\Delta(p-1)}\left(\rho(a), \lambda_{1}\right)=0
\end{aligned}
$$

and then,

$$
(p-1)\left(\lambda_{1}+\lambda_{2}\right) \int_{\rho(a)}^{b} y_{1}^{\sigma(p-1)}\left(t, \lambda_{1}\right) y_{2}^{\sigma(p-1)}\left(t, \lambda_{2}\right) \Delta t-2(p-1) \int_{\rho(a)}^{b} r(t) y_{1}^{\sigma(p-1)}\left(t, \lambda_{1}\right) y_{2}^{\sigma(p-1)}\left(t, \lambda_{2}\right) \Delta t=0,
$$

for $\lambda_{1} \neq \lambda_{2}$. Hence, it shows that the eigenfunctions $y_{1}^{(p-1)}\left(t, \lambda_{1}\right), y_{2}^{(p-1)}\left(t, \lambda_{2}\right)$ are always orthogonal. 
Theorem 4. The equality

$$
\begin{aligned}
& y^{(p-1)}(t, \lambda) \frac{\partial}{\partial \lambda} y^{\Delta(p-1)}(t, \lambda)-y^{\Delta(p-1)}(t, \lambda) \frac{\partial}{\partial \lambda} y^{(p-1)}(t, \lambda) \\
& =-2 \lambda(p-1) \int_{\rho(a)}^{t}\left(y^{\sigma(p-1)}(\tau, \lambda)\right)^{2} \Delta \tau+2(p-1) \int_{\rho(a)}^{t} r(\tau)\left(y^{\sigma(p-1)}(\tau, \lambda)\right)^{2} \Delta \tau,
\end{aligned}
$$

holds where $t \in[\rho(a), b] \cap \mathbb{T}$ and $\lambda \in \mathbb{R}$ for the problem (1)-(3).

Theorem 5. All eigenvalues of the problem (1)-(3) are real.

Proof. Let $\bar{\lambda}_{0}$ be a complex eigenvalue and $\bar{y}^{(p-1)}(t)$ be an eigenfunction corresponding to the eigenvalue $\bar{\lambda}_{0}$ of the problem (1)-(3). Then, we obtain

$$
\begin{aligned}
& \left\{y^{\Delta(p-1)} \bar{y}^{(p-1)}-\bar{y}^{\Delta(p-1)} y^{(p-1)}\right\}^{\Delta} \\
& =(p-1)\left(\bar{\lambda}_{0}^{2}-\lambda_{0}^{2}\right)\left|y^{\sigma(p-1)}\right|^{2}-2(p-1)\left(\bar{\lambda}_{0}-\lambda_{0}\right)\left|y^{\sigma(p-1)}\right|^{2} .
\end{aligned}
$$

If we use $\Delta$-integral for the last equality from $\rho(a)$ to $b$, and by considering the boundary conditions (2), (3), we get

$$
(p-1)\left(\bar{\lambda}_{0}+\lambda_{0}\right) \int_{\rho(a)}^{b}\left|y^{\sigma(p-1)}(t)\right|^{2} \Delta t-2(p-1) \int_{\rho(a)}^{b} r(t)\left|y^{\sigma(p-1)}(t)\right|^{2} \Delta t=0 \Rightarrow y^{\sigma(p-1)}(t)=0,
$$

for $\bar{\lambda}_{0} \neq \lambda_{0}$ and $\left(\bar{\lambda}_{0}+\lambda_{0}\right)-2 r(t)>0$. This is a contradiction and the proof is completed.

Now, we will prove Picone's identity which is an important formula for the problem (1)-(3) to prove oscillation criteria. There are many studies about the Picone's identity (see [22-26]). In 1998, Allegretto and Xi [27] obtained a Picone's identity for the $p$-Laplace operator and $\mathrm{Bal}[28]$ showed a generalized Picone's identity for the $p$-Laplace operator and then proved Sturmian comparison principle and a Liouville type theorem (see also [2931]). Picone's identity for dynamic equations on time scales was studied in [32-34].

Let denote the kernel of the matrix $A$ by $\operatorname{Ker} A$, and put $\operatorname{def} M=\operatorname{dim} \operatorname{Ker} A$, and $\alpha^{\diamond}=1 / \alpha$ for $\alpha \neq 0$ and $0^{\diamond}=0$. It is given below Picone's identity for the problem (1)-(3).

Theorem 6. Let $\operatorname{Ker} y^{(p-1)}(t) \subset \operatorname{Ker} x^{(p-1)}(t)$ and $\operatorname{Ker} y^{\sigma(p-1)}(t) \subset \operatorname{Ker} x^{\sigma(p-1)}(t)$, $x^{(p-1)} \in C_{r d}^{2}([\rho(a), b], \mathbb{R})$ and $y^{(p-1)}$ is a solution of $L y^{(p-1)}+\lambda^{2}(1-p) y^{\sigma(p-1)}=0$.

Then,

$$
\begin{aligned}
& \left\{L\left(x^{(p-1)}\right)-\lambda^{2}(p-1) x^{\sigma(p-1)}\right\} x^{\sigma(p-1)} \\
& =\left[x^{(p-1)}\left(y^{(p-1)}\right)^{\diamond} W\left(x^{(p-1)}, y^{(p-1)}\right)\right]^{\Delta}+\left(y^{(p-1)} y^{\sigma(p-1)}\right)^{\diamond} W^{2}\left(x^{(p-1)}, y^{(p-1)}\right),
\end{aligned}
$$

where $W\left(x^{(p-1)}, y^{(p-1)}\right)=x^{(p-1)} y^{\Delta(p-1)}-y^{(p-1)} x^{\Delta(p-1)}$.

Proof. Suppose that $\left(y^{(p-1)} y^{\sigma(p-1)}\right)(t) \neq 0$. Then, we get 


$$
\begin{aligned}
& {\left[x^{(p-1)}\left(y^{(p-1)}\right)^{\diamond} W\left(x^{(p-1)}, y^{(p-1)}\right)\right]^{\Delta}=\frac{x^{\sigma(p-1)}}{y^{\sigma(p-1)}} W^{\Delta}\left(x^{(p-1)}, y^{(p-1)}\right)} \\
& +\left(\frac{x^{\Delta(p-1)} y^{(p-1)}-x^{(p-1)} y^{\Delta(p-1)}}{y^{(p-1)} y^{\sigma(p-1)}}\right) W\left(x^{(p-1)}, y^{(p-1)}\right) \\
& =\frac{x^{\sigma(p-1)}}{y^{\sigma(p-1)}}\left\{-L\left(y^{(p-1)}\right) x^{\sigma(p-1)}+L\left(x^{(p-1)}\right) y^{\sigma(p-1)}\right\}-\frac{W^{2}\left(x^{(p-1)}, y^{(p-1)}\right)}{y^{(p-1)} y^{\sigma(p-1)}} \\
& =\frac{x^{\sigma(p-1)}}{y^{\sigma(p-1)}}\left\{-\lambda^{2}(p-1) y^{\sigma(p-1)} x^{\sigma(p-1)}+L\left(x^{(p-1)}\right) y^{\sigma(p-1)}\right\}-\frac{W^{2}\left(x^{(p-1)}, y^{(p-1)}\right)}{y^{(p-1)} y^{\sigma(p-1)}} \\
& =\left\{L\left(x^{(p-1)}\right)-\lambda^{2}(p-1) x^{\sigma(p-1)}\right\} x^{\sigma(p-1)}-\left(y^{(p-1)} y^{\sigma(p-1)}\right)^{\diamond} W^{2}\left(x^{(p-1)}, y^{(p-1)}\right) .
\end{aligned}
$$

If $t$ is right-scattered and $\left(y^{(p-1)} y^{\sigma(p-1)}\right)(t)=0$, then $\left(x^{(p-1)} x^{\sigma(p-1)}\right)(t)=0$ and

$$
\begin{aligned}
& W\left(x^{(p-1)}, y^{(p-1)}\right)=x^{(p-1)} y^{\Delta(p-1)}-y^{(p-1)} x^{\Delta(p-1)} \\
& =x^{(p-1)}\left(y^{\sigma(p-1)}-y^{(p-1)}\right) / \mu-y^{(p-1)}\left(x^{\sigma(p-1)}-x^{(p-1)}\right) / \mu \\
& =\left(x^{(p-1)} y^{\sigma(p-1)}-y^{(p-1)} x^{\sigma(p-1)}\right) / \mu=0 .
\end{aligned}
$$

If $t$ is left-scattered, $x^{(p-1)}\left(y^{(p-1)}\right)^{\diamond} W\left(x^{(p-1)}, y^{(p-1)}\right)$ is continuous at $t$. If $t$ is left dense, $x^{(p-1)}\left(y^{(p-1)}\right)^{\diamond} W\left(x^{(p-1)}, y^{(p-1)}\right)$ is also continuous at $t$. By using L'Hospital rule, we get

$$
\begin{aligned}
\left.\lim _{s \rightarrow t^{-}}\left[x^{(p-1)}\left(y^{(p-1)}\right)^{\diamond} W\left(x^{(p-1)}, y^{(p-1)}\right)\right](s)=\frac{x^{\Delta(p-1)}(t)}{y^{\Delta(p-1)}(t)} W^{\Delta}\left(x^{(p-1)}, y^{(p-1)}\right)\right]=0 \\
=\left[x^{(p-1)}\left(y^{(p-1)}\right)^{\diamond} W\left(x^{(p-1)}, y^{(p-1)}\right)\right](t) . \\
\left.x^{(p-1)}\left(y^{(p-1)}\right)^{\diamond} W\left(x^{(p-1)}, y^{(p-1)}\right)\right]^{\Delta} \\
=\frac{\left[x^{(p-1)}\left(y^{(p-1)}\right)^{\diamond} W\left(x^{(p-1)}, y^{(p-1)}\right)\right]^{\sigma}-x^{(p-1)}\left(y^{(p-1)}\right)^{\diamond} W\left(x^{(p-1)}, y^{(p-1)}\right)}{\mu} \\
=\frac{\left[x^{(p-1)}\left(y^{(p-1)}\right)^{\diamond} W\left(x^{(p-1)}, y^{(p-1)}\right)\right]^{\sigma}}{\mu}=x^{\sigma(p-1)}\left(y^{\sigma(p-1)}\right)^{\diamond} W^{\Delta}\left(x^{(p-1)}, y^{(p-1)}\right) \\
=x^{\sigma(p-1)}\left(y^{\sigma(p-1)}\right)^{\diamond}\left\{-L\left(y^{(p-1)}\right) x^{\sigma(p-1)}+L\left(x^{(p-1)}\right) y^{\sigma(p-1)}\right\} \\
=\frac{x^{\sigma(p-1)}}{y^{\sigma(p-1)}}\left\{-\lambda^{2}(p-1) y^{\sigma(p-1)} x^{\sigma(p-1)}+L\left(x^{(p-1)}\right) y^{\sigma(p-1)}\right\} \\
=\left\{L\left(x^{(p-1)}\right)-\lambda^{2}(p-1) x^{\sigma(p-1)}\right\} x^{\sigma(p-1)}-\left(y^{(p-1)} y^{\sigma(p-1)}\right)^{\diamond} W^{2}\left(x^{(p-1)}, y^{(p-1)}\right) .
\end{aligned}
$$

If $t$ is right-dense point, the Picone's identity holds.

\section{Conclusion}

$p$ - Laplacian type Diffusion eigenvalue problems arise from many fields of science and have various applications in mathematics and physics. We notice that much attention paid on discussing the solutions for this type problems for different operators on time 
scales. Hence, we have considered some spectral properties for $p$-Laplacian Diffusion eigenvalue problem on an arbitrary time scale and generalized basic results. We have obtained some important results to fill the gaps in this area.

\section{References}

1. S. Hilger, Ph.D. Thesis, Universitat Wurzburg, (1988)

2. M. Bohner, Boston(MA), Birkhauser, Boston Inc, (2001)

3. R. Agarwal, M. Bohner, D. O'Regan, A Peterson, J. Comput. Appl. Math., 141, 1$26(2002)$

4. G.Sh. Guseinov, B. Kaymakçalan, J. Comput. Appl. Math., 141, 187-196 (2002)

5. L. Erbe, A. Peterson, P. Řehák, J. Math. Anal. Appl., 275, 418-438 (2002)

6. G.Sh. Guseinov, J. Math. Anal. Appl., 285, 107-127 (2003)

7. B.P. Rynne, J. Math. Anal. Appl., 328, 1217-1236 (2007)

8. S.R. Grace, M. Bohner, R.P. Agarwal, J. Difference Equ. Appl., 15, 451-460 (2009)

9. C. Song, P. Weng, Nonlinear Analy. Theory, 68, 208-215 (2008)

10. W. Han, S. Kang,. Math. Comput. Model., 49, 527-535 (2009)

11. J. R. Graef, L. Kong, J. Difference Equ. Appl., 17, 831-839 (2011).

12. A. Dogan, Adv. Difference Equ., 2013, 238 (2013).

13. D. Anderson, R. Avery, J. Henderson, J. Difference Equ. Appl., 10, 889-896 (2004)

14. Z. He, J. Comput. Appl. Math., 182, 304-315 (2005)

15. H.R. Sun, W.T. Li, J. Differential Equations, 240, 217-248 (2007).

16. P. Binding, P.J. Browne, I.M. Karabash, Proc. Edinb. Math. Soc., 53, 271-291 (2010)

17. Y.H. Su, Z. Feng, Appl. Anal., 1-19 (2017)

18. H. Koyunbakan, Bound. Value Probl., 2013, 8 pp. (2013)

19. T. Gulsen, E. Yilmaz, Commun. Fac. Sci. Univ. Ank. Sér. A1 Math. Stat., 65, 23$36(2016)$

20. T. Gulsen, S.S.M. Sian, E. Yilmaz, H. Koyunbakan, Int. J. Anal. Appl., 16, $137-$ $148(2018)$

21. M.G. Gasymov, G.Sh. Guseinov, Dokl. Akad. Nauk Az. SSSR, 37, 19-23 (1981)

22. K. Kreith, , J. Math. Anal. Appl., 31, 297-308 (1970)

23. C.A. Swanson, Picone's identity, Rend. Mat, 8, 373-397 (1975)

24. G.J. Etgen, , R.T. Lewis, Proc. Edinb. Math. Soc., 22, 277-290 (1979)

25. D. Hinton, Birkhäuser Basel. pp. 1-27 (2005)

26. T. Kusano, J. Jaroš, N. Yoshida, Nonlinear Analysis: Theory, Methods and Applications, 40, 381-395 (2000)

27. W. Allegretto, H.Y. Xi, Nonlinear Analy. Theory, 32, 819-830 (1998)

28. K. Bal, Electron. J. Differ. Equ., 2013, 1-6 (2013)

29. X. Yang, Appl. Math. Comput., 136, 181-193 (2003)

30. A. Tiryaki, Electronic J. Differential Equations, 2016, 1-7 (2016)

31. O. Došlý, S. Hilger, J. Comput. Appl. Math., 141, 147-158 (2002)

32. B. Belinskiy, J.R. Graef, S. Petrovic. Int. J. Difference Equ., 2, 25-35 (2007)

33. D.R., Anderson, J.R. Graef, Appl. Anal. Discrete Math., 4, 338-346 (2010)

34. R.P. Agarwal, M. Bohner, P. J. Wong, Appl. Math. Comput., 99, 153-166 (1999) 